\title{
Optical spectroscopy of GaN microcavities with thicknesses controlled using a plasma etchback
}

\author{
R. W. Martin ${ }^{\text {a) }}$ and P. R. Edwards \\ Department of Physics and Applied Physics, University of Strathclyde, Glasgow G4 ONG, Scotland, \\ United Kingdom

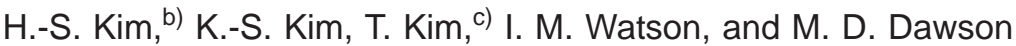 \\ Institute of Photonics, University of Strathclyde, Glasgow G4 ONW, Scotland, United Kingdom \\ Y. Cho and T. Sands \\ Department of Materials Science and Engineering, University of California, Berkeley, California 94720
}

N. W. Cheung

Department of Electrical Engineering and Computer Sciences, University of California, Berkley, California 94720-1772

(Received 8 June 2001; accepted for publication 27 August 2001)

\begin{abstract}
The effect of an etch-back step to control the cavity length within GaN-based microcavities formed between two dielectric Bragg mirrors was investigated using photoluminescence and reflectivity. The structures are fabricated using a combination of a laser lift-off technique to separate epitaxial III-N layers from their sapphire substrates and electron-beam evaporation to deposit silica/zirconia multilayer mirrors. The photoluminescence measurements reveal cavity modes from both etched and nonetched microcavities. Similar cavity finesses are measured for 2.0 and $0.8 \mu \mathrm{m} \mathrm{GaN}$ cavities fabricated from the same wafer, indicating that the etchback has had little effect on the microcavity quality. For InGaN quantum well samples the etchback is shown to allow controllable reduction of the cavity length. Two etch steps of $100 \mathrm{~nm}$ are demonstrated with an accuracy of approximately $5 \%$. The etchback, achieved using inductively coupled plasma and wet chemical etching, allows removal of the low-quality GaN nucleation layer, control of the cavity length, and modification of the surface resulting from lift-off. (C) 2001 American Institute of Physics.
\end{abstract}

[DOI: $10.1063 / 1.1415769$ ]

Building on the successful development of highperformance AlGaInN light-emitting diodes (LEDs) and laser diodes, attention is now being directed towards other IIInitride optical devices including short-wavelength vertical cavity surface-emitting lasers (VCSELs). ${ }^{1-3}$ Device processing issues are very important for the realization of these, and related, AlGaInN devices. One approach to III-nitride VCSELs involves the fabrication of both mirrors from all-oxide distributed Bragg reflectors (DBRs), ${ }^{2,4,5}$ which possess a number of advantages over their semiconductor based counterparts. In this approach critical issues include achieving a suitably thin cavity and being able to control its thickness. Ideally a device would employ resonant periodic gain with InGaN quantum wells positioned at antinodes of the cavity electric field. For III-nitride microcavities this may be achieved by controllable postgrowth thinning of a GaN buffer layer after separation from its substrate. A further benefit of such processing is the removal of the poor quality GaN nucleation layers generally found at the substrate interface. In this letter, we demonstrate the suitability of inductively coupled plasma (ICP) etching for precise control of the

\footnotetext{
${ }^{a}$ Electronic mail: r.w.martin@strath.ac.uk

${ }^{b}$ Presently at Samsung Electronics, Suwon, Korea.

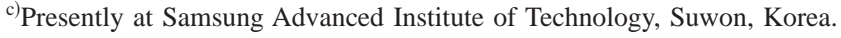

cavity length in GaN based microcavities fabricated using two oxide DBR mirrors and substrate removal by laser liftoff (LLO).

The III-nitride heterostructures were fabricated by metalorganic chemical vapor deposition in an Aixtron 200 series reactor using sapphire (0001) substrates. After initial experiments using a single layer of GaN, approximately $2 \mu \mathrm{m}$ thick, single and multiple InGaN quantum wells were introduced. The wells, designed for blue emission, were grown following deposition of a $\mathrm{GaN}$ buffer layer, approximately $1 \mu \mathrm{m}$ thick, and capped with $10 \mathrm{~nm}$ of undoped GaN. A 10 $\frac{1}{2}$ pair $\mathrm{SiO}_{2} / \mathrm{ZrO}_{2}$ stack was then deposited on the top $\mathrm{GaN}$ surface by electron beam evaporation. The surface of this mirror is bonded to a $\mathrm{Si}$ wafer and the sapphire substrate was removed by irradiation with a $\mathrm{KrF}$ laser $(248 \mathrm{~nm})$ followed by melting of the thin Ga-rich interfacial layer. ${ }^{6}$ A second all-oxide DBR mirror is then deposited onto this surface, creating a microcavity. Alternatively, prior to the second mirror deposition, an etch-back step is included in order to remove the low quality GaN nucleation layer, control the microcavity length, and modify the surface of the GaN. Before depositing the second mirror the exposed $\mathrm{GaN}$ surface is chemically cleaned using aqueous hydrochloric acid to remove contaminants including elemental gallium.

The etch was performed using inductively coupled $\mathrm{Cl}_{2} / \mathrm{BCl}_{3} / \mathrm{Ar}$ plasmas. Details of the ICP etch conditions are described elsewhere. ${ }^{7}$ Variation of the $\mathrm{Cl}_{2}: \mathrm{BCl}_{3}$ ratio, with a 



FIG. 1. SEM images of GaN microcavities formed using all-oxide mirrors and laser liftoff (a) without ICP etchback and (b) after ICP etch back, reducing the GaN thickness from $2 \mu \mathrm{m}$ to approximately $800 \mathrm{~nm}$.

constant $20 \%$ of argon in the gas mixture, showed that the smoothest surface was achieved with a $24 \% \mathrm{BCl}_{3}$ mixture. The root mean square (rms) surface roughness, measured by atomic force microscopy on a "large" $(20 \mu \mathrm{m} \times 20 \mu \mathrm{m})$ area, was $\sim 15 \mathrm{~nm}$. This corresponded to an ICP etch rate of $480 \mathrm{~nm} / \mathrm{min}$, slightly below the maximum obtained for LLO $\mathrm{GaN}(550 \mathrm{~nm} / \mathrm{min})$.

The structure and surface morphologies of the resulting cavities were observed by scanning electron microscopy (SEM). Figure 1 shows cross-sectional SEM images of a nonetched and an etched GaN layer sandwiched by two alloxide DBRs. In both cases the surface views show that some of the roughness is in the form of pits, which are believed to result from the explosive decomposition of the GaN characteristic of the LLO process.

The optical properties of the GaN microcavities were measured by reflectance and photoluminescence (PL) spectra. A spectrophotometer was used for the reflectance and the PL employed a $325 \mathrm{~nm} \mathrm{HeCd}$ laser as the excitation source and a cooled charge coupled device (CCD) detection system
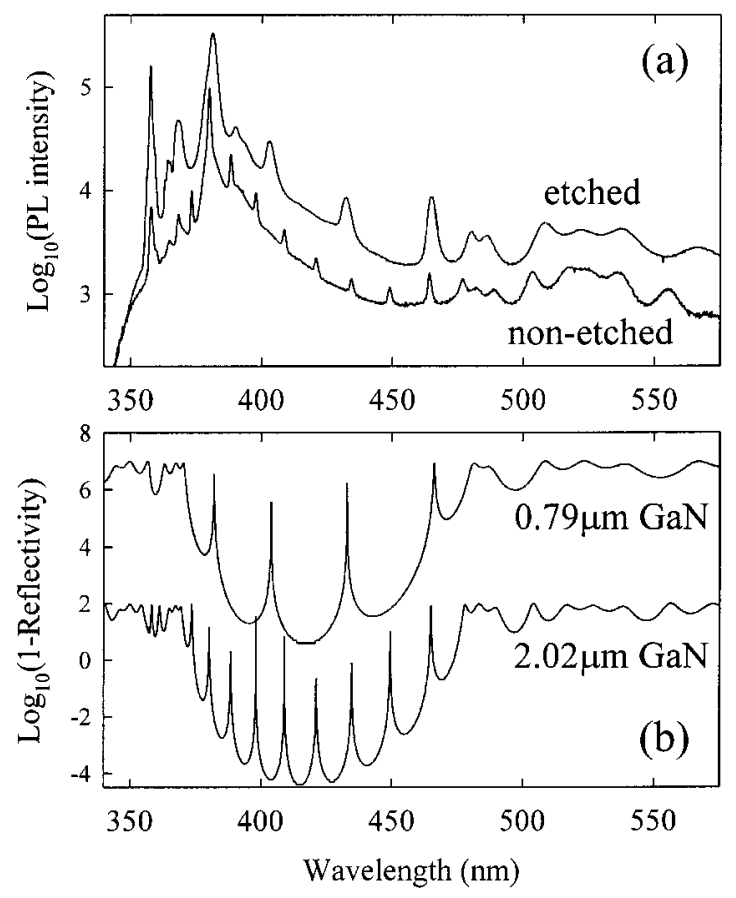

FIG. 2. (a) Measured PL spectra ( $\sim 15 \mathrm{~K})$ from etched and nonetched GaN microcavities. (b) Simulated reflectivity data for structures with different thickness $\mathrm{GaN}$ layers between two $10 \frac{1}{2}$ period $\mathrm{SiO}_{2} / \mathrm{ZrO}_{2}$ mirrors. The

traces in (b) were offset for clarity.
Downloaded 11 Jan 2011 to 130.159 .17 .136 . Redistribution subject to AIP license or copyright; see http://apl.aip.org/about/rights_and_permissions


FIG. 3. Room temperature reflectivity and photoluminescence data (solid lines) from unetched microcavity samples containing (a) a single InGaN quantum well and (b) a 10 period multiple InGaN quantum well. The dashed line in (a) shows room temperature PL from the as-grown quantum well wafer.

(0.2 $\mathrm{nm}$ resolution). Both excitation and collection of the PL were performed through the "top" mirror (the side of the original substrate). Figure 2(a) shows low temperature PL spectra from the two GaN microcavities shown in Fig. 1. A number of sharp cavity modes are resolved in the wavelength range of $370-470 \mathrm{~nm}$, which corresponds approximately to the high-reflectivity stop band of the DBR mirrors. Figure 2(b) shows results of simulations of the reflectivity spectra for $\mathrm{GaN}$ layers sandwiched by two $10 \frac{1}{2}$ period silica/zirconia mirrors centered at $420 \mathrm{~nm}$. Room temperature refractive index data are obtained from Refs. 8-10, with the reduction upon cooling computed where necessary. ${ }^{11}$ Excellent agreement for the spectral position of the cavity modes is obtained using $\mathrm{GaN}$ thicknesses of 2.02 and $0.79 \mu \mathrm{m}$ for the nonetched and etched samples. These thicknesses agree with those in the SEM images, as expected. When considering the shape of the spectra in Fig. 2(a) it is important to note that the $\mathrm{GaN}$ luminescence is dominated by the characteristic band-edge and donor-acceptor pair transitions in the wavelength region of 355-390 nm, with very little emission in the region of 390-470 nm that corresponds to the bulk of the mirror stop band. The peak widths [full width at half maximum (FWHM)] are approximately 3.0 and $1.3 \mathrm{~nm}$ for the etched and nonetched samples, respectively, giving finesse of approximately 10 in both cases. Thus there is no evidence of any detrimental effects of the etchback on the quality of the mirrors or the PL.

While important for giving details of the cavity's structural quality the optical data from these samples are limited by the small amount of GaN luminescence within the reflectivity stop band. Room temperature PL and reflectivity data from similar microcavities containing blue-emitting single and multiple InGaN quantum wells are shown in Figs. 3 and 4. In these cases the second DBR was not ideal, as shown by the reflectivity spectra, but in all structures fabricated a single sharp mode was observed in both the reflectivity and PL spectra. The degradation of the mirror is thought to relate to some remaining surface contamination and improved chemical cleaning prior to deposition should recover the thecense or copyright; see http://apl.aip.org/about/rights_and_permissions 




FIG. 4. Room temperature photoluminescence data from single quantum well structures with (a) no etchback, (b) nominal $100 \mathrm{~nm}$ etchback, and (c) nominal $200 \mathrm{~nm}$ etchback. The arrows show the mode positions calculated assuming a single layer of GaN, of the thickness stated, between two $10 \frac{1}{2}$ period DBR mirrors.

oretical flat-topped reflectivity stop band, as was achieved for the structures in Figs. 1 and 2. Figure 3(a) includes results from the nonetched single quantum well, showing the PL FWHM narrowing from $13.4 \mathrm{~nm}$ for the as-grown quantum well to $0.6 \mathrm{~nm}$ for the microcavity sample, indicating cavity finesse of $\sim 50$ and resonance quality factor, $Q$, of $\sim 750$, similar to that reported in Ref. 2 . This finesse corresponds to a reflectivity of $\sim 97 \%$ for each of two identical mirrors. Figure 3(b) shows data from a multiple quantum well cavity sample.

The overall thickness of the as-grown samples is in the region of $1 \mu \mathrm{m}$ and nonetched samples are compared with those in which the target of the etchback was to remove 100 and $200 \mathrm{~nm}$ of GaN. Figure 4 shows luminescence data from the single quantum well (SQW), for which ex situ reflectometry gave a total nitride thickness of $0.90 \mu \mathrm{m}$. The cavity mode is observed to shift to shorter wavelength, and to broaden, with an increased amount of etchback. The wavelength of the cavity mode depends very strongly on the cavity length, with a $3 \mathrm{~nm}$ change in the latter producing a spectral shift of about $1 \mathrm{~nm}$ for a given mode. Simulations of the cavity reflectivity therefore allow an estimation of the amount of material removed by etching. Assuming a single layer of $\mathrm{GaN}$ between $10 \frac{1}{2}$ period silica/zirconia mirrors centered at $420 \mathrm{~nm}$ gives a thickness of $898 \mathrm{~nm}$ for the unetched SQW sample, in excellent agreement with the ex situ reflectometry. This thickness is reduced by 104 and $212 \mathrm{~nm}$ for the nominal 100 and $200 \mathrm{~nm}$ etch-back structures, respectively.
Note that a different mode is observed in each case. The largest source of error here will arise from uncertainties in the refractive index data. The variation in PL peak position around the sample surface is typically in the region of $\pm 1 \mathrm{~nm}$. Given the limited amount of calibration for the etch process these values indicate that a very high degree of control of the cavity length is possible. Furthermore the shifts illustrated in Fig. 4 indicate how selective area etching could be used to produce emission at a range of wavelengths from a single wafer. The broadening of the PL peaks, and increasing background, does indicate some degradation of the sample quality with etching in these quantum well samples. It is anticipated that further chemical cleaning of the surfaces following ICP etching could remedy this, at least partially.

In summary, photoluminescence data from III-N cavities, fabricated using laser liftoff and two dielectric DBRs, demonstrate cavity filtered luminescence. The use of an etchback step to reduce the cavity length by over $1 \mu \mathrm{m}$ in $\mathrm{GaN}$ cavity samples is not seen to reduce the finesse. For thinner samples containing InGaN quantum wells a single strong mode is seen in the cavity filtered PL. Controllable ICP etching of the GaN prior to mirror deposition is shown to allow the cavity length to be accurately tailored to a specific value.

The financial support of UK EPSRC is gratefully acknowledged. The authors also thank C. H. Jeoung, D. H. Lee, and Professor G. Y. Yeom of Sungkyunkwan University for atomic force microscopy data.

${ }^{1}$ T. Someya, K. Tachibana, J. Lee, T. Kamiya, and Y. Arakawa, Jpn. J. Appl. Phys., Part 2 37, L1424 (1998).

${ }^{2}$ Y.-K. Song, H. Zhou, M. Diagne, A. V. Nurmikko, R. P. Schneider, C. P. Kuo, M. R. Krames, R. S. Kern, C. Carter-Coman, and F. A. Kish, Appl. Phys. Lett. 76, 1662 (2000).

${ }^{3}$ I. L. Krestnikov, W. V. Lundin, A. V. Sakharov, V. A. Semenov, A. S. Usikov, A. F. Tsatsul'nikov, Zh. I. Alferov, N. N. Ledentsov, A. Høffmann, and D. Bimberg, Appl. Phys. Lett. 75, 1192 (1999).

${ }^{4}$ T. Kim, R. W. Martin, I. M. Watson, M. D. Dawson, T. F. Krauss, J. H. Marsh, and R. M. De La Rue, Mater. Sci. Eng., B 82, 245 (2001).

${ }^{5}$ R. W. Martin, P. R. Edwards, R. Pecharroman-Gallego, C. Trager-Cowan, T. Kim, H.-S. Kim, K.-S. Kim, I. M. Watson, M. D. Dawson, T. F. Krauss, J. H. Marsh, and R. M. De La Rue, Phys. Status Solidi A 183, 145 (2001).

${ }^{6}$ W. S. Wong, Y. Cho, E. R. Weber, T. Sands, K. M. Yu, J. Kruger, A. B. Wengrow, and N. W. Cheung, Appl. Phys. Lett. 75, 1887 (1999).

${ }^{7}$ H. S. Kim, T. Kim, K. S. Kim, I. M. Watson, R. W. Martin, J. H. Marsh, R. M. De La Rue, and M. D. Dawson, Proceedings of International Workshop on Nitride Semiconductors 2000 (Institute of Pure and Applied Physics, Tokyo, 2000), pp. 750-753.

${ }^{8}$ T. Peng and J. Piprek, Electron. Lett. 32, 2285 (1996).

${ }^{9}$ Handbook of Optics, Edited by M. Bass (McGraw-Hill, New York, 1995).

${ }^{10}$ V. Craciun, D. Craciun, and I. W. Boyd, Electron. Lett. 34, 1527 (1998).

${ }^{11}$ D. Brunner, H. Angerer, E. Bustarret, F. Freudenberg, R. Hopler, R. Dimitrov, O. Ambacher, and M. Stutzmann, J. Appl. Phys. 82, 5090 (1997). 